\title{
El rol del cirujano general en una unidad de cuidados respiratorios en la pandemia de 2020
}

\section{The role of the general surgeon in a respiratory care unit in the 2020 pandemic}

\author{
Erwin I. Marín-Pardo*, Elías Ballesteros-Suárez, Guadalupe K. Peña-Portillo, Sandra G. Ayala-Hernández, \\ Delfino H. Pérez-Cervantes y Guadalupe del C. Pérez-Torres
}

Departamento de Cirugía General, Hospital Universitario de Puebla, Puebla, Pue., México

El 31 de diciembre de 2019 la Comisión Municipal de Salud de Wuhan (provincia de Hubei, China) notificó un conglomerado de casos de neumonía en la ciudad. Posteriormente se determinó que estaban causados por un nuevo coronavirus ${ }^{1}$. El 27 de febrero de 2020 se confirmó el primer caso en México ${ }^{2}$ y el 11 de marzo de 2020 la Organización Mundial de la Salud (OMS) declaró que la COVID-19 (coronavirus disease 2019) puede caracterizarse como una pandemia ${ }^{3}$.

A casi 12 meses, más de 53.7 millones de casos confirmados y más de 1.3 millones de muertes ${ }^{4}$, la COVID-19 ha cambiado la forma en que se entendía el mundo actual. Los sistemas de salud mundiales han sido rebasados, las consecuencias sociales, económicas y políticas han puesto en jaque incluso a las potencias mundiales. A pesar de los esfuerzos del ensayo "Solidaridad" y de las múltiples investigaciones que se están llevando a cabo, seguimos sin encontrar una terapia completamente efectiva y el lograr inmunizar a la población mundial parece aún lejano.

En el ámbito quirúrgico, las investigaciones se han centrado en el riesgo de la cirugía en pacientes infectados. Se ha concluido que las complicaciones pulmonares postoperatorias ocurren en la mitad de los pacientes con infección perioperatoria por SARSCoV-2 (severe acute respiratory syndrome coronavirus 2) y están asociadas con una alta mortalidad, sugiriendo así la necesidad de posponer procedimientos no urgentes y promover tratamientos no quirúrgicos para retrasar o evitar la necesidad de cirugía ${ }^{5}$. Asimismo, se trató sobre la necesidad de realizar adecuaciones a los quirófanos, la suspensión de la consulta externa, el equipo de protección personal, las modificaciones al consentimiento informado y más aspectos que tratan sobre la seguridad de los pacientes y del personal quirúrgico. Ojalá eso fuera el centro de la discusión, ojalá la pandemia no hubiera golpeado $\tan$ fuerte.

En México, como en la mayoría de los países en vías de desarrollo, la pandemia ha traído consecuencias devastadoras, un sistema de salud históricamente olvidado no fue capaz de contener la brutal embestida de la pandemia. Esto ha obligado a la reconversión hospitalaria, desde los más grandes institutos hasta los centros de salud, la atención de pacientes infectados por SARS-CoV-2 se ha vuelto el punto medular de la salud pública en México, y esto ha llevado no solo a la reconversión hospitalaria, sino también a la reconversión del personal de la salud.

Inicialmente, la primera línea de defensa hospitalaria para la atención de estos pacientes fue encabezada por médicos intensivistas, neumólogos, internistas e infectólogos, entre otros, pero no hay suficientes especialistas en México capaces de atender al número de
Correspondencia:

*Erwin I. Marín-Pardo

E-mail: imarpar_19@ hotmail.es
Fecha de recepción: 25-11-2020

Fecha de aceptación: 03-08-2021

DOI: 10.24875/RHJM.20000122
Disponible en internet: 17-12-2021 Rev Hosp Jua Mex. 2020;88(4):161-163

www.revistahospitaljuarez.com 1405-9622/@ 2021 Sociedad Médico-Quirúrgica del Hospital Juárez de México, A.C. Publicado por Permanyer. Este es un artículo open access bajo la licencia CC BY-NC-ND (http://creativecommons.org/licenses/by-nc-nd/4.0/). 
enfermos que día a día se suman a la larga lista de pacientes hospitalizados con cuadros de infección grave. Aunado a esto, el personal en contacto con estos pacientes finalmente ha ido enfermando, y en algunos muy lamentables casos, muriendo, lo que fue mermando la ya de por sí limitada cantidad de médicos calificados para atender a estos enfermos.

Así pues, muchos hospitales en México se han visto en la necesidad de echar mano de médicos de otras áreas para la atención en unidades de cuidados respiratorios, y el cirujano general, por su experiencia en el tratamiento del paciente séptico, chocado, en el manejo de líquido y electrolitos, en los cuidados del paciente postoperado, además de las habilidades propias de la especialidad para la realización de procedimientos invasivos y quirúrgicos, se ha vuelto un elemento ideal para formar parte de la segunda línea de defensa de atención. De tal forma que pasamos de preocuparnos sobre cómo adecuar nuestro quirófano a ocuparnos sobre decidir la terapia más adecuada para el paciente con neumonía que ahora está a nuestro cargo, pasamos de tratar al paciente con sepsis de origen abdominal por el de sepsis de origen pulmonar, pasamos de formar parte de un equipo quirúrgico a un equipo multidisciplinario perfectamente organizado que trabaja codo a codo para tratar a pacientes graves con infección por SARS-CoV-2, síndrome de dificultad respiratoria aguda y todas las alteraciones orgánicas que de estos surgen.

Este equipo, improvisado pero capaz y dispuesto, formado por médicos intensivistas, internistas, anestesiólogos y ahora cirujanos generales que se encuentran de lleno en la unidad de cuidados respiratorios, cumplen con roles puntualmente asignados, con los que se pretende brindar una atención integral a los pacientes enfermos, pudiendo tener en un mismo escenario a un anestesiólogo intubando, un intensivista reanimando y a un cirujano colocando un catéter venoso central y una sonda endopleural, ganando así tiempo para el paciente y disminuyendo la posibilidad de errores que pudieran derivar en complicaciones médicas y en contagios entre el personal.

Este modelo de atención es prueba de la capacidad de los cirujanos generales para la atención del paciente críticamente enfermo y de la adaptabilidad con la que los médicos mexicanos hemos sido capaces de responder de acuerdo con el contexto social en México.

Que esta pandemia sirva como parteaguas para que los gobiernos se sensibilicen sobre la importancia de fortalecer la infraestructura, recursos humanos y materiales de la salud en México. Queda para la reflexión: que ante escenarios tan sombríos como este, el trabajo en equipo es más fuerte que cualquier individualidad y que el bien común va más allá de las diferencias que entre especialidades pueda haber; para los hospitales formadores de residentes la necesidad de seguir formando cirujanos generales y especialistas capaces de atender al paciente críticamente enfermo; para las universidades la necesidad de crear planes de estudio que se adapten a los cambios del panorama de salud mundial actual, y para la población en general la urgencia por modificar hábitos del estilo de vida que nos lleven a ser una sociedad más sana, que nos haga menos vulnerable a esta y muchas otras enfermedades.

Los días pasan y el escenario se complica cada vez más, diversas ciudades en el mundo se han visto en la necesidad de cerrar sus fronteras, y en México no se descarta la posibilidad de que un escenario similar se pueda presentar. Médicos de todas las áreas se encuentran dedicando todos sus esfuerzos para luchar contra la COVID-19. Vamos a ganar, a pesar de todas las adversidades y de lo largo que parezca el camino, vamos a ganar, y mientras dure, los cirujanos vamos a estar ahí.

\section{Agradecimientos}

Los autores agradecen al personal de la unidad de cuidados respiratorios del Hospital Universitario de Puebla que día a día dan lo mejor de ellos para atender a los pacientes enfermos de COVID-19.

\section{Financiamiento}

Los autores declaran que no se recibió ningún tipo de apoyo financiero para la realización de este trabajo.

\section{Conflicto de intereses}

Los autores declaran que no existe ningún conflicto de intereses.

\section{Responsabilidades éticas}

Protección de personas y animales. Los autores declaran que para esta investigación no se han realizado experimentos en seres humanos ni en animales.

Confidencialidad de los datos. Los autores declaran que en este artículo no aparecen datos de pacientes.

Derecho a la privacidad y consentimiento informado. Los autores declaran que en este artículo no aparecen datos de pacientes. 


\section{Bibliografía}

1. World Health Organization. Pneumonia of unknown cause - China [Internet]. World Health Organization; 5 de enero de 2020 [consultado: 20 de julio de 2020). Disponible en: https://www.who.int/csr/don/05-january2020-pneumonia-of-unkown-cause-china/es

2. Gobierno de México. Se confirma en México caso importado de coronavirus COVID-19 [Internet]. Gobierno de México; 28 de febrero de 2020 [consultado: 20 de julio de 2020]. Disponible en: https://www.gob.mx/ salud/prensa/077-se-confirma-en-mexico-caso-importado-de-coronavirus-covid-19
3. World Health Organization. Timeline of WHO's response to COVID-19 [Internet]. World Health Organization; 29 de junio de 2020 [consultado: 20 de julio de 2020]. Disponible en: https://www.who.int/news-room/detail/29-06-2020-covidtimeline

4. World Health Organization. Weekly epidemiological update - 17 November 2020 [Internet]. World Health Organization; 17 de noviembre de 2020 [consultado: 19 de noviembre de 2020]. Disponible en: https://www.who. int/docs/default-source/coronaviruse/situation-reports/20200719-covid-19-sitrep-181.pdf?sfvrsn=82352496_2

5. Nepogodiev D, Bhangu A, Glasbey JC, Li E, Omar OM, Simoes JFF, et al. Mortality and pulmonary complications in patients undergoing surgery with perioperative SARS-CoV-2 infection: an international cohort study. Lancet. 2020;396:27-38. 